\title{
Rotational effects on the negative magnetic pressure instability
}

\author{
I. R. Losada ${ }^{1,2}$, A. Brandenburg ${ }^{3,4}$, N. Kleeorin ${ }^{5,3}$, D. Mitra ${ }^{3}$, and I. Rogachevskii ${ }^{5,3}$ \\ 1 Department of Astrophysics, Universidad de La Laguna, 38206 La Laguna (Tenerife), Spain \\ e-mail: illa.rivero.losada@gmail.com \\ 2 Instituto de Astrofísica de Canarias, C/ Vía Láctea, s/n, La Laguna, Tenerife, Spain \\ 3 Nordita, Royal Institute of Technology and Stockholm University, Roslagstullsbacken 23, 10691 Stockholm, Sweden \\ ${ }^{4}$ Department of Astronomy, AlbaNova University Center, Stockholm University, 10691 Stockholm, Sweden \\ 5 Department of Mechanical Engineering, Ben-Gurion University of the Negev, POB 653, 84105 Beer-Sheva, Israel
}

Received 23 July 2012 / Accepted 21 September 2012

\begin{abstract}
Context. The surface layers of the Sun are strongly stratified. In the presence of turbulence with a weak mean magnetic field, a largescale instability resulting in the formation of nonuniform magnetic structures, can be excited on the scale of many (more than ten) turbulent eddies (or convection cells). This instability is caused by a negative contribution of turbulence to the effective (mean-field) magnetic pressure and has previously been discussed in connection with the formation of active regions.

Aims. We want to understand the effects of rotation on this instability in both two and three dimensions.

Methods. We use mean-field magnetohydrodynamics in a parameter regime in which the properties of the negative effective magnetic pressure instability have previously been found to agree with properties of direct numerical simulations.

Results. We find that the instability is already suppressed for relatively slow rotation with Coriolis numbers (i.e. inverse Rossby numbers) around 0.2. The suppression is strongest at the equator. In the nonlinear regime, we find traveling wave solutions with propagation in the prograde direction at the equator with additional poleward migration away from the equator.

Conclusions. We speculate that the prograde rotation of the magnetic pattern near the equator might be a possible explanation for the faster rotation speed of magnetic tracers relative to the plasma velocity on the Sun. In the bulk of the domain, kinetic and current helicities are negative in the northern hemisphere and positive in the southern.
\end{abstract}

Key words. magnetohydrodynamics (MHD) - hydrodynamics - turbulence - dynamo

\section{Introduction}

In the outer parts of the Sun, energy is transported through turbulent convection. The thermodynamic aspects of this process are well understood through mixing length theory (Vitense 1953). Also reasonably well understood is the partial conversion of kinetic energy into magnetic energy via dynamo action (Parker 1979; Zeldovich et al. 1983). Most remarkable is the possibility of generating magnetic fields on much larger spatial and temporal scales than the characteristic turbulence scales. This has now been seen in many three-dimensional turbulence simulations (Brandenburg 2001; Brandenburg \& Subramanian 2005), but the physics of this is best understood in terms of mean-field theory, which encapsulates the effects of complex motions in terms of effective equations for mean flow and mean magnetic field (Moffatt 1978; Parker 1979; Krause \& Rädler 1980).

The effects of stratification are usually only included to leading order and often only in connection with rotation, because the two together give rise to the famous $\alpha$ effect, which is able to explain the generation of large-scale magnetic fields (Krause \& Rädler 1980). In recent years, however, a completely different effect arising from strong stratification alone has received attention: the suppression of turbulent pressure by a weak mean magnetic field. This effect mimics a negative effective (mean-field) magnetic pressure owing to a negative contribution of turbulence to the mean magnetic pressure. Under suitable conditions, this leads to the negative effective magnetic pressure instability (NEMPI), which can cause the formation of magnetic flux concentrations. In turbulence simulations, this instability has only been seen recently (Brandenburg et al. 2011), because significant scale separation is needed to overcome the effects of turbulent diffusion (Brandenburg et al. 2012). Mean-field considerations, however, have predicted the existence of NEMPI for a long time (Kleeorin et al. 1989, 1990, 1996; Kleeorin \& Rogachevskii 1994; Rogachevskii \& Kleeorin 2007; Brandenburg et al. 2010).

One of the remarkable insights is that NEMPI can occur at any depth, depending just on the value of the mean magnetic field strength. However, for a domain of given depth the instability can only occur in the location where the dependence of effective turbulent pressure on the ratio of field strength to equipartition value has a negative slope. Once this is obeyed, the only other necessary condition for NEMPI to occur is that the turbulent diffusivity is low enough. In practice this means that there are enough turbulent eddies within the domain of investigation (Brandenburg et al. 2012; Kemel et al. 2012c).

Despite the potential importance of NEMPI, many additional effects have not yet been explored. The idea is that NEMPI would interact with the global dynamo producing the large-scale magnetic field for NEMPI to act upon. Thus, the field needs to be self-consistently generated. Ideally, global geometry is needed, and such calculations should be three-dimensional (3D), because one expects flux concentrations not to be two-dimensional (2D) or axisymmetric. New mean-field coefficients will appear in such a more general case, and not much is known about them. Nevertheless, although other terms may appear, it will be interesting to investigate the evolution of NEMPI in more realistic cases with just the leading term responsible for the instability. 
The goal of the present paper is to include the effects of rotation in NEMPI in a local Cartesian domain at a given latitude in the Sun. To this end we determine the dependence of growth rate and saturation level of NEMPI on rotation rate and latitude, and to characterize rotational effects on the resulting flux concentrations. We restrict ourselves to a mean-field treatment and denote averaged quantities by an overbar. Furthermore, we make the assumption of an isothermal equation of state. This is of course quite unrealistic, as far as applications to the Sun are concerned. However, it has been found earlier that NEMPI has similar properties both for an isothermal layer with an isothermal equation of state and a nearly isentropic one with the more general perfect gas law (Käpylä et al. 2012). Given that our knowledge of NEMPI is still rather limited, it is useful to consider the new effects of rotation within the framework of the conceptually simpler case of an isothermal layer.

We begin with the model equations, discuss the linear theory of NEMPI in the presence of rotation, and consider 2D and 3D numerical models.

\section{The model}

We consider here an isothermal equation of state with constant sound speed $c_{\mathrm{s}}$, so the mean gas pressure is $\bar{p}=\bar{\rho} c_{\mathrm{s}}^{2}$. The evolution equations for mean velocity $\overline{\boldsymbol{U}}$, mean density $\bar{\rho}$, and mean vector potential $\bar{A}$, are

$$
\begin{aligned}
& \frac{\mathrm{D} \overline{\boldsymbol{U}}}{\mathrm{D} t}=-2 \boldsymbol{\Omega} \times \overline{\boldsymbol{U}}-c_{\mathrm{s}}^{2} \boldsymbol{\nabla} \ln \bar{\rho}+\boldsymbol{g}+\overline{\mathcal{F}}_{\mathrm{M}}+\overline{\mathcal{F}}_{\mathrm{K}}, \\
& \frac{\mathrm{D} \bar{\rho}}{\mathrm{D} t}=-\bar{\rho} \boldsymbol{\nabla} \cdot \overline{\boldsymbol{U}} \\
& \frac{\partial \overline{\boldsymbol{A}}}{\partial t}=\overline{\boldsymbol{U}} \times \overline{\boldsymbol{B}}-\left(\eta_{\mathrm{t}}+\eta\right) \overline{\boldsymbol{J}}
\end{aligned}
$$

where $\mathrm{D} / \mathrm{D} t=\partial / \partial t+\overline{\boldsymbol{U}} \cdot \boldsymbol{\nabla}$ is the advective derivative, $\eta_{\mathrm{t}}$ and $\eta$ are turbulent and microscopic magnetic diffusivities, $\boldsymbol{g}=(0,0,-g)$ is the acceleration due to the gravity field,

$\overline{\mathcal{F}}_{\mathrm{K}}=\left(v_{\mathrm{t}}+v\right)\left(\nabla^{2} \overline{\boldsymbol{U}}+\frac{1}{3} \boldsymbol{\nabla} \boldsymbol{\nabla} \cdot \overline{\boldsymbol{U}}+2 \overline{\mathbf{S}} \boldsymbol{\nabla} \ln \bar{\rho}\right)$

is the total (turbulent plus microscopic) viscous force with $v_{\mathrm{t}}$ being the turbulent viscosity, and $\overline{\mathrm{S}}_{i j}=\frac{1}{2}\left(\bar{U}_{i, j}+\bar{U}_{j, i}\right)-\frac{1}{3} \delta_{i j} \boldsymbol{\nabla} \cdot \overline{\boldsymbol{U}}$ is the traceless rate of strain tensor of the mean flow. The mean Lorentz force, $\overline{\mathcal{F}}_{\mathrm{M}}$, is given by

$\bar{\rho} \overline{\mathcal{F}}_{\mathrm{M}}=\overline{\boldsymbol{J}} \times \overline{\boldsymbol{B}}+\frac{1}{2} \nabla\left(q_{\mathrm{p}} \overline{\boldsymbol{B}}^{2}\right)$,

where $\overline{\boldsymbol{J}}=\boldsymbol{\nabla} \times \overline{\boldsymbol{B}} / \mu_{0}$ the mean current density, $\mu_{0}$ is the vacuum permeability, and the last term, $\frac{1}{2} \nabla\left(q_{\mathrm{p}} \overline{\boldsymbol{B}}^{2}\right)$, on the righthand side of Eq. (5) determines the turbulent contribution to the mean Lorentz force. Following Brandenburg et al. (2012) and Kemel et al. (2012a), the function $q_{\mathrm{p}}(\beta)$ is approximated by:

$q_{\mathrm{p}}(\beta)=\frac{\beta_{\star}^{2}}{\beta_{\mathrm{p}}^{2}+\beta^{2}}$,

where $\beta_{\star}$ and $\beta_{\mathrm{p}}$ are constants, $\beta=\bar{B} / B_{\text {eq }}$ is the modulus of the normalized mean magnetic field, and $B_{\text {eq }}=\sqrt{\mu_{0} \rho} u_{\mathrm{rms}}$ the equipartition field strength. The angular velocity vector $\boldsymbol{\Omega}$ is quantified by its scalar amplitude $\Omega$ and colatitude $\theta$, such that

$\boldsymbol{\Omega}=\Omega(-\sin \theta, 0, \cos \theta)$.

(7) $\frac{\mathrm{D} \bar{B}_{y}}{\mathrm{D} t}=-\bar{B}_{y} \boldsymbol{\nabla} \cdot \overline{\boldsymbol{U}}$,
In this arrangement, $z$ corresponds to radius, $x$ to colatitude, and $y$ to azimuth.

Following the simplifying assumption of recent direct numerical simulations of NEMPI (Brandenburg et al. 2011), we assume that the root-mean-square turbulent velocity, $u_{\mathrm{rms}}$, is constant in space and time. For an isothermal density stratification,

$\bar{\rho}=\rho_{0} \exp \left(-z / H_{\rho}\right)$,

where $H_{\rho}=c_{\mathrm{s}}^{2} / g$ is the density scale height, we then have $B_{\text {eq }}(z)$. To quantify the strength of the imposed field, we also define $B_{\text {eq0 }}=B_{\text {eq }}(z=0)$. The value of $u_{\mathrm{rms}}$ is also related to the values of $\eta_{\mathrm{t}}$ and $v_{\mathrm{t}}$, which we assume to be equal, with $\eta_{\mathrm{t}}=v_{\mathrm{t}}=$ dies of the underlying turbulence. This formula assumes that the relevant correlation time is $\left(u_{\mathrm{rms}} k_{\mathrm{f}}\right)^{-1}$, which has been shown to be fairly accurate (Sur et al. 2008).

\section{Linear theory of NEMPI with rotation}

In this section we study the effect of rotation on the growth rate of NEMPI. Following earlier work (e.g., the appendix of Kemel et al. 2012c), and for simplicity, we neglect dissipation processes, use the anelastic approximation, $\boldsymbol{\nabla} \cdot \bar{\rho} \overline{\boldsymbol{U}}=0$, and assume that the density scale height $H_{\rho}=$ const. We consider the equation of motion, ignoring the $\bar{U} \cdot \nabla \bar{U}$ nonlinearity,

$\frac{\partial \overline{\boldsymbol{U}}(t, x, z)}{\partial t}=-2 \boldsymbol{\Omega} \times \overline{\boldsymbol{U}}-\frac{1}{\bar{\rho}} \boldsymbol{\nabla} p_{\mathrm{tot}}+\boldsymbol{g}$,

where $p_{\text {tot }}=\bar{p}+p_{\text {eff }}$ is the total pressure consisting of the sum of the mean gas pressure $\bar{p}$, and the effective magnetic pressure, $p_{\text {eff }}=\left(1-q_{\mathrm{p}}\right) \bar{B}^{2} / 2$, where $\bar{B}=|\overline{\boldsymbol{B}}|$. Here and elsewhere the vacuum permeability is set to unity. We assume for simplicity that $\partial_{y}=0$, and that the mean magnetic field only has a $y$-component, $\overline{\boldsymbol{B}}=\left(0, \bar{B}_{y}(x, z), 0\right)$, so the mean magnetic tension, $\overline{\boldsymbol{B}} \cdot \boldsymbol{\nabla} \overline{\boldsymbol{B}}$ in Eq. (9) vanishes.

Taking twice the curl of Eq. (9), and noting further that $\hat{z}$. $\boldsymbol{\nabla} \times \boldsymbol{\nabla} \times \overline{\boldsymbol{U}}=-\Delta \bar{U}_{z}+\nabla_{z} \boldsymbol{\nabla} \cdot \overline{\boldsymbol{U}}$, we obtain

$$
\begin{aligned}
\frac{\partial}{\partial t}\left[\Delta \bar{U}_{z}+\nabla_{z}(\overline{\boldsymbol{U}} \cdot \boldsymbol{\nabla} \ln \bar{\rho})\right]=-2 \boldsymbol{\Omega} \cdot \boldsymbol{\nabla}(\boldsymbol{\nabla} \times \overline{\boldsymbol{U}})_{z} \\
+\nabla_{x}\left[\left(\nabla_{z} \frac{p_{\text {tot }}}{\bar{\rho}}\right) \frac{\nabla_{x} \bar{\rho}}{\bar{\rho}}-\left(\nabla_{x} \frac{p_{\text {tot }}}{\bar{\rho}}\right) \frac{\nabla_{z} \bar{\rho}}{\bar{\rho}}\right],
\end{aligned}
$$

where we have used the anelastic approximation in the form $\boldsymbol{\nabla} \cdot \overline{\boldsymbol{U}}=-\overline{\boldsymbol{U}} \cdot \boldsymbol{\nabla} \ln \bar{\rho}$ and the fact that under the curl the gradient can be moved to $\bar{\rho}$. We have also taken into account that $\Omega_{y}=0$ and have used Eq. (30) of Kemel et al. (2012c) to relate the double curl of $\left(\nabla p_{\text {tot }}\right) / \bar{\rho}$ to the last term in Eq. (10). The first term on the righthand side of Eq. (10) for $\bar{U}_{z}$ is proportional to $(\boldsymbol{\nabla} \times \overline{\boldsymbol{U}})_{z}$. Taking the $z$ component of the curl of Eq. (9) we obtain the following equation for $(\boldsymbol{\nabla} \times \overline{\boldsymbol{U}})_{z}$ :

$\frac{\partial}{\partial t}(\boldsymbol{\nabla} \times \overline{\boldsymbol{U}})_{z}=2\left(\boldsymbol{\Omega} \cdot \boldsymbol{\nabla}-\frac{\Omega_{z}}{H_{\rho}}\right) \bar{U}_{z}$.

The induction equation for $\bar{B}_{y}(x, z)$ is given by $u_{\mathrm{rms}} / 3 k_{\mathrm{f}}$, where $k_{\mathrm{f}}$ is the wavenumber of the energy-carrying ed- 
where $\mathrm{D} / \mathrm{D} t=\partial / \partial+\overline{\boldsymbol{U}} \cdot \boldsymbol{\nabla}$ is the advective derivative. For a magnetic field with only a $y$-component, but $\partial / \partial y=0$, there is no stretching term, so there is no term of the form $\overline{\boldsymbol{B}} \cdot \boldsymbol{\nabla} \overline{\boldsymbol{U}}$.

We linearize Eqs. (10)-(12), indicating small changes by $\delta$. We consider an equilibrium with a constant magnetic field of the form $\left(0, B_{0}, 0\right)$, a zero mean velocity, and the fluid density as given by Eq. (8). We take into account that the function $q_{\mathrm{p}}=$ $q_{\mathrm{p}}(\beta)$ depends both on $\bar{B}$ and on $\bar{\rho}$, which implies that (Kemel et al. 2012c)

$\delta\left(\frac{p_{\text {tot }}}{\bar{\rho}}\right)=\frac{1}{2} v_{\mathrm{A}}^{2}\left(1-q_{\mathrm{p}}-\frac{\mathrm{d} q_{\mathrm{p}}}{\mathrm{d} \ln \beta^{2}}\right)\left(2 \frac{\delta \bar{B}_{y}}{B_{0}}-\frac{\delta \bar{\rho}}{\bar{\rho}}\right)$,

while

$\nabla_{z}\left(\frac{p_{\text {tot }}}{\bar{\rho}}\right)=\frac{1}{2} v_{\mathrm{A}}^{2}\left(1-q_{\mathrm{p}}-\frac{\mathrm{d} q_{\mathrm{p}}}{\mathrm{d} \ln \beta^{2}}\right) \frac{1}{H_{\rho}}$.

The linearized system of equations reads as

$$
\begin{aligned}
\frac{\partial}{\partial t}\left(\Delta-\frac{1}{H_{\rho}} \nabla_{z}\right) \delta \bar{U}_{z} & =2 \frac{v_{\mathrm{A}}^{2}}{H_{\rho}} \frac{\mathrm{d} \mathcal{P}_{\mathrm{eff}}}{\mathrm{d} \beta^{2}} \frac{\nabla_{x}^{2} \delta \bar{B}_{y}}{B_{0}}-2 \boldsymbol{\Omega} \cdot \boldsymbol{\nabla}(\boldsymbol{\nabla} \times \delta \overline{\boldsymbol{U}})_{z} \\
\frac{\partial}{\partial t}(\boldsymbol{\nabla} \times \delta \overline{\boldsymbol{U}})_{z} & =2\left(\boldsymbol{\Omega} \cdot \boldsymbol{\nabla}-\frac{\Omega_{z}}{H_{\rho}}\right) \delta \bar{U}_{z} \\
\frac{\partial \delta \bar{B}_{y}}{\partial t} & =-B_{0} \frac{\delta \bar{U}_{z}}{H_{\rho}}
\end{aligned}
$$

where $\mathcal{P}_{\text {eff }}(\beta)=\frac{1}{2}\left[1-q_{\mathrm{p}}(\beta)\right] \beta^{2}$ is the effective magnetic pressure normalized by the local value of $B_{\text {eq }}^{2}$.

Introducing a new variable $V_{z}=\sqrt{\bar{\rho}} \delta \bar{U}_{z}$ in Eqs. (15)-(17) and after simple transformations we arrive at the following equation for one variable $V_{z}$ :

$\frac{\partial^{2}}{\partial t^{2}}\left(\Delta-\frac{1}{4 H_{\rho}^{2}}\right) V_{z}+\left((2 \boldsymbol{\Omega} \cdot \boldsymbol{\nabla})^{2}-\frac{\Omega_{z}^{2}}{H_{\rho}^{2}}\right) V_{z}=\lambda_{0}^{2} \nabla_{x}^{2} V_{z}$,

where

$\lambda_{0}^{2}(z)=-2 \frac{v_{\mathrm{A}}^{2}(z)}{H_{\rho}^{2}} \frac{\mathrm{d} \mathcal{P}_{\mathrm{eff}}(z)}{\mathrm{d} \beta^{2}}$.

In the WKB approximation, which is valid when $k_{z} H_{\rho} \gg 1$, i.e., when the characteristic scale of the spatial variation of the perturbations of the magnetic and velocity fields are much smaller than the density height length, $H_{\rho}$, the growth rate of the largescale instability (NEMPI) is given by

$\lambda=\left[\lambda_{0}^{2} \frac{k_{x}^{2}}{k^{2}}-\omega_{\text {inert }}^{2}\right]^{1 / 2}$,

where $\omega_{\text {inert }}=2 \boldsymbol{\Omega} \cdot \hat{\boldsymbol{k}}$ is the frequency of the inertial waves. Here, $\hat{\boldsymbol{k}}=\boldsymbol{k} / \boldsymbol{k}$ is the unit vector of $\boldsymbol{k}$. A necessary condition for the instability is

$$
\frac{\mathrm{d} \mathcal{P}_{\text {eff }}}{\mathrm{d} \beta^{2}}<0 \text {. }
$$

NEMPI can be excited even in a uniform mean magnetic field, and the source of free energy of the instability is provided by the small-scale turbulence. In contrast, the free energy in Parker's magnetic buoyancy instability (Parker 1966) or in the interchange instability (Tserkovnikov 1960; Priest 1982) is drawn from the gravitational field. Both instabilities are excited in a plasma when the characteristic scale of variations in the original horizontal magnetic field is smaller than the density scale height. As seen from Eq. (20), $\lambda$ is either real or purely imaginary, so no complex eigenvalues are possible, as would be required for growing oscillatory solutions.

Without rotation the growth rate of NEMPI is (Kleeorin et al. 1993; Rogachevskii \& Kleeorin 2007; Kemel et al. 2012d)

$\lambda=\lambda_{0} \frac{k_{x}}{k}$.

The rotation reduces the growth rate of NEMPI, which can be excited when $k_{x} / k>\omega_{\text {inert }} / \lambda_{0}$ and $\mathrm{d} \mathcal{P}_{\text {eff }} / \mathrm{d} \beta^{2}<0$. In the opposite case, $k_{x} / k<\omega_{\text {inert }} / \lambda_{0}$, the large-scale instability is not excited, while the frequency of the inertial waves is reduced by the effective negative magnetic pressure.

For an arbitrary vertical inhomogeneity of the density, we seek a solution to Eq. (18) in the form $V_{z}(t, x, z)=V(z) \exp (\lambda t+$ $\left.\mathrm{i} k_{x} x\right)$ and obtain an eigenvalue problem

$\left[\nabla_{z}^{2}+\frac{8 \Omega_{x} \Omega_{z}}{\lambda^{2}+4 \Omega_{z}^{2}} \mathrm{i} k_{x} \nabla_{z}-\Lambda^{2} k_{x}^{2}-\frac{1}{4 H_{\rho}^{2}}\right] V(z)=0$,

where

$\Lambda^{2}=\frac{\lambda^{2}-\lambda_{0}^{2}(z)+4 \Omega_{x}^{2}}{\lambda^{2}+4 \Omega_{z}^{2}}$,

and $\lambda$ is the eigenvalue. Equation (23) can be reduced to the Schrödinger type equation, $\Psi^{\prime \prime}-\tilde{U}(R) \Psi=0$, via the transformation

$\begin{aligned} \Psi(R) & =\sqrt{R} V(z) \exp \left(\mathrm{i} \frac{4 \Omega_{x} \Omega_{z}}{\lambda^{2}+4 \Omega_{z}^{2}} k_{x} z\right), \\ R(z) & =\frac{v_{A 0}^{2}}{u_{\mathrm{rms}}^{2} \beta_{\mathrm{p}}^{2}} \mathrm{e}^{z / H_{\rho}},\end{aligned}$

where $v_{\mathrm{A} 0}=B_{0} / \sqrt{\bar{\rho}_{0}}$ is the Alfvén speed based on the averaged density, the potential $\tilde{U}(R)$ is

$\tilde{U}(R)=\frac{k_{x}^{2} H_{\rho}^{2}}{R\left(\lambda^{2}+4 \Omega_{z}^{2}\right)}\left[\frac{\lambda^{2}}{R}\left(\frac{\lambda^{2}+4 \Omega^{2}}{\lambda^{2}+4 \Omega_{z}^{2}}\right)+\frac{u_{\mathrm{rms}}^{2} \beta_{\mathrm{p}}^{2}}{H_{\rho}^{2}}\left(1-\frac{q_{\mathrm{p} 0}}{(1+R)^{2}}\right)\right]$,

and we have used Eq. (6) for $q_{\mathrm{p}}$ with $\beta_{\star}=\beta_{\mathrm{p}} \sqrt{q_{\mathrm{p} 0}}$ and $q_{\mathrm{p} 0}=$ $q_{\mathrm{p}}(\beta=0)$. As follows from Eq. (27), the potential, $\tilde{U}(R)$, is positive for $R \rightarrow 0$ and $R \rightarrow \infty$. Therefore, for the existence of the instability, the potential should have a negative minimum. This is possible when $q_{\mathrm{p} 0}>(1+R)^{2}$. When the potential $\tilde{U}(R)$ has a negative minimum, there are two points $R_{1}$ and $R_{2}$ (the socalled turning points) in which $\tilde{U}\left(R=R_{1,2}\right)=0$. Figure 1 shows $\tanh \tilde{U}(R)$ for different values of $\Omega$. This representation allows us to distinguish the behavior for low values of $\tilde{U}(R)$.

Using Eq. (27) and the condition $\tilde{U}\left(R=R_{1,2}\right)=0$, we estimate the maximum growth rate of the instability as

$\lambda=\frac{1}{\sqrt{2}}\left[\lambda_{*}^{2}-4 \Omega^{2}+\left[\left(\lambda_{*}^{2}-4 \Omega^{2}\right)^{2}+8 \Omega_{z}^{2} \lambda_{*}^{2}\right]^{1 / 2}\right]^{1 / 2}$,

where

$\lambda_{*}=\frac{\beta_{\star} u_{\mathrm{rms}}}{H_{\rho}} \frac{\left[R_{1} R_{2}\left(2+R_{1}+R_{2}\right)\right]^{1 / 2}}{\left(1+R_{1}\right)\left(1+R_{2}\right)}$. 


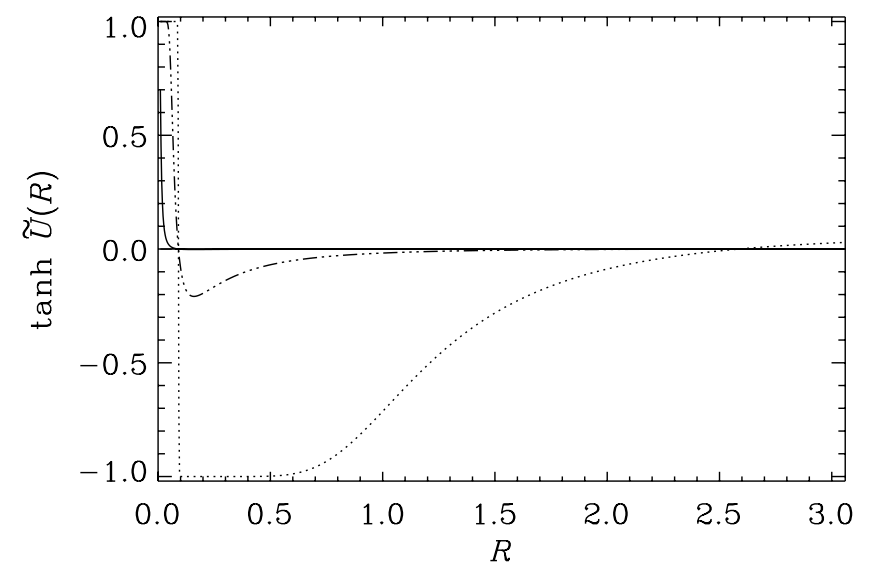

Fig. 1. $\tanh \tilde{U}(R)$ for $\tilde{\lambda} \equiv \lambda / \lambda_{*}=0.02, \theta=0$, and $\Omega=0.01$ (dotted line), 0.1 (dashed-dotted line), and 1 (solid line).

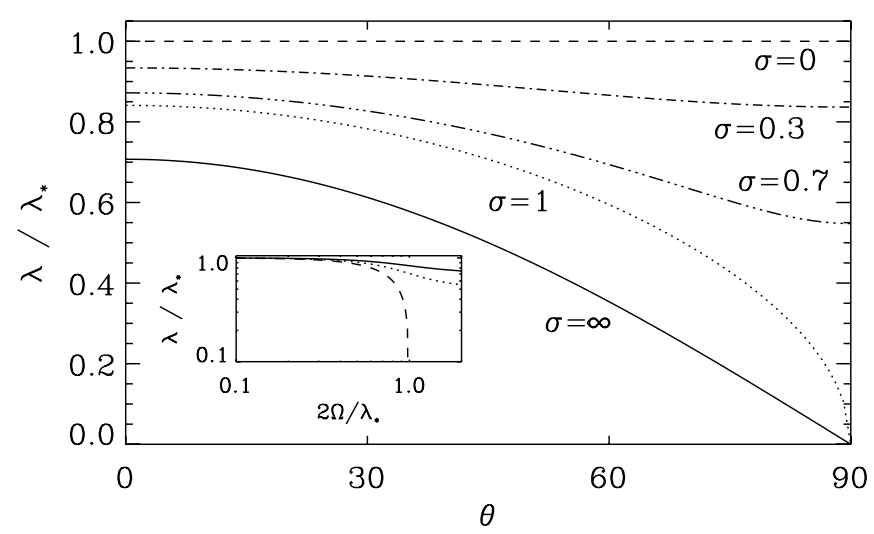

Fig. 2. Theoretical dependence of $\lambda / \lambda_{*}$ on $\theta$ for different values of $\sigma$ using Eq. (30). The inset shows the dependence of $\lambda / \lambda_{*}$ on $2 \Omega / \lambda_{*}=$ $\sigma^{1 / 2}$ for $\theta=0^{\circ}$ (solid), $45^{\circ}$ (dotted), and $90^{\circ}$ (dashed).

By defining $\sigma=4 \Omega^{2} / \lambda_{*}^{2}$, Eq. (28) can also be written as

$\lambda / \lambda_{*}=\frac{1}{\sqrt{2}}\left[1-\sigma+\left(1-2 \sigma \sin ^{2} \theta+\sigma^{2}\right)^{1 / 2}\right]^{1 / 2}$.

For $\sigma \gg 1$, we obtain $\lambda / \lambda_{*}=\cos \theta / \sqrt{2}$, which is independent of the value of $\sigma$. In Fig. 2 we plot the dependence of $\lambda / \lambda_{*}$ on $\theta$ for different values of $\sigma$ and on $2 \Omega / \lambda_{*}=\sigma^{1 / 2}$ for different values of $\theta$ (inset).

Unfortunately, the asymptotic analysis does not allow full information about the system. Therefore we turn in the following to numerical simulations of the full $2 \mathrm{D}$ and $3 \mathrm{D}$ mean-field equations.

\section{Numerical results}

In this section we discuss numerical mean-field modeling. We consider computational domains of size $L^{2}$ or $L^{3}$ with periodic boundary conditions in the horizontal direction(s) and stress-free perfect conductor boundary conditions in the vertical direction. The smallest wavenumber that fits horizontally into the domain has the wavenumber $k_{1}=2 \pi / L$. The numerical simulations are performed with the PENCIL CODE ${ }^{1}$, which uses sixth-order explicit finite differences in space and a third-order accurate time stepping method (Brandenburg \& Dobler 2002). As units of length we use $k_{1}^{-1}$, and time is measured in units of $\left(c_{\mathrm{s}} k_{1}\right)^{-1}$.

\footnotetext{
1 http://pencil-code.googlecode.com
}

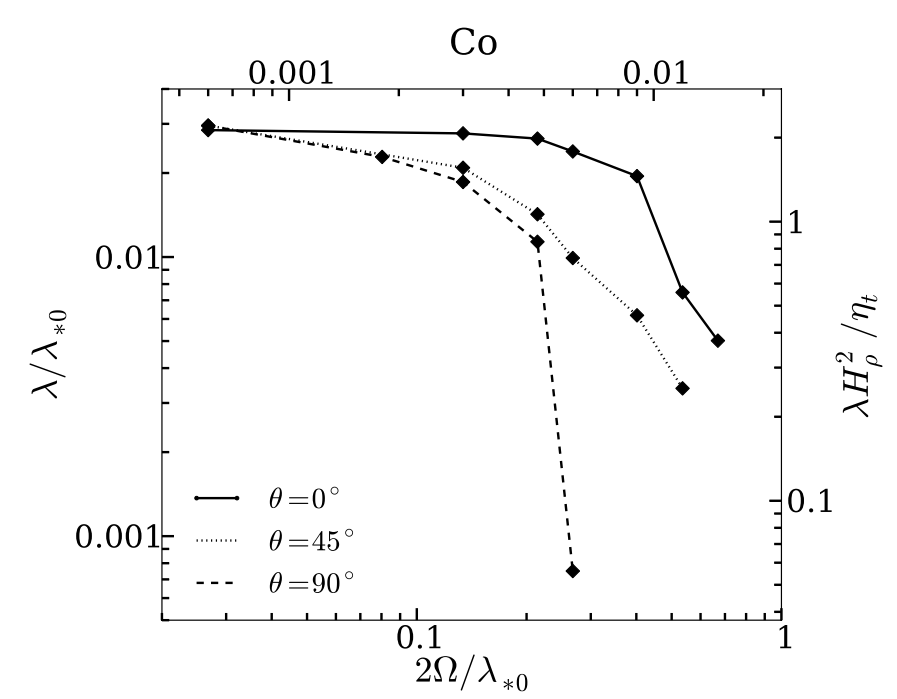

Fig. 3. Dependence of $\lambda / \lambda_{* 0}$ on $2 \Omega / \lambda_{* 0}$ for three values of $\theta$ for $2 \mathrm{D}$ simulations with $B_{0} / B_{\text {eq } 0}=0.1$.

An important nondimensional parameter is the Coriolis number, Co $=2 \Omega / u_{\mathrm{rms}} k_{\mathrm{f}}$. Using $k_{\mathrm{f}}=u_{\mathrm{rms}} / 3 \eta_{\mathrm{t}}$, we can express this in terms of the parameter $C_{\Omega}=\Omega / \eta_{\mathrm{t}} k_{1}^{2}$, which is often used in mean-field dynamo theory. Thus, we have

$\mathrm{Co}=6 \eta_{\mathrm{t}} \Omega / u_{\mathrm{rms}}^{2}=6\left(\eta_{\mathrm{t}} k_{1} / u_{\mathrm{rms}}\right)^{2} C_{\Omega}$.

Motivated by the analytic results of the previous section we normalize the growth rate of the instability alternatively by a quantity $\lambda_{* 0} \equiv \beta_{\star} u_{\mathrm{rms}} / H_{\rho}$. In the following we take $u_{\mathrm{rms}} / c_{\mathrm{s}}=0.1$. Furthermore, we use $v_{\mathrm{t}}=\eta_{\mathrm{t}}=10^{-3} c_{\mathrm{s}} / k_{\mathrm{f}}$, so that $k_{\mathrm{f}} H_{\rho} \approx 33$ and $\eta_{\mathrm{t}} k_{1} / u_{\mathrm{rms}}=10^{-2}$. This also means that for $\Omega=0.01$, for example, we have $2 \Omega / \lambda_{* 0}=0.27$ and $\mathrm{Co}=0.006$.

For the models presented below, we use $q_{\mathrm{p} 0}=20$ and $\beta_{\mathrm{p}}=0.167$, which corresponds to $\beta_{\star}=0.75$, and is appropriate for the parameter regime in which $R_{\mathrm{m}} \approx 18$ and $k_{\mathrm{f}} / k_{1}=30$ (Kemel et al. $2012 \mathrm{~d}$ ). We use either $B_{0} / B_{\text {eq } 0}=0.1$ or 0.05 . We recall, however, that the growth rate does not depend on this choice, provided the bulk of the eigenfunction fits into the domain, which is the case here for both values of $B_{0}$. For the lower value of $B_{0}$ the maximum of the magnetic structures (i.e., the maximum of the eigenfunction in $z$ ) is slightly higher up in the domain, but in both cases the maximum is contained within the domain.

We discuss first the $\Omega$ and $\theta$ dependence of $2 \mathrm{D}$ and $3 \mathrm{D}$ solutions. Using $\theta=0^{\circ}, 45^{\circ}$, and $90^{\circ}$, corresponding to $90^{\circ}, 45^{\circ}$, and $0^{\circ}$ latitude, we find that NEMPI is suppressed for rotation rates around $\Omega \approx 0.01 c_{\mathrm{s}} k_{1}$ and 0.025 in $2 \mathrm{D}$ and $3 \mathrm{D}$, as can be seen in Figs. 3 and 4. This corresponds to Co $=0.006$ and 0.015 , which are remarkably low values. We note a similar behavior in 2D and 3D: NEMPI is suppressed for even lower values of $2 \Omega / \lambda_{* 0}$ as $\theta$ increases. Moreover, there is qualitative agreement between the results of mean-field simulations and the predictions based on asymptotic analysis, even though in the former case we normalized by $\lambda_{* 0}$, while in the latter we normalized by $\lambda_{*}$; see Eq. (30).

Next, we vary $\theta$. As expected from the results of Sect. 3, and as already seen in Figs. 3 and 4, the largest growth rates occur at the poles $\left(\theta=0^{\circ}\right)$, and NEMPI is the most strongly suppressed at the equator. The growth rate as a function of $\theta$ is given in Fig. 5 for two values of $2 \Omega / \lambda_{* 0}$, showing a minimum at $\theta=90^{\circ}$ (i.e., at the equator). In the upper panel of Fig. 5, 


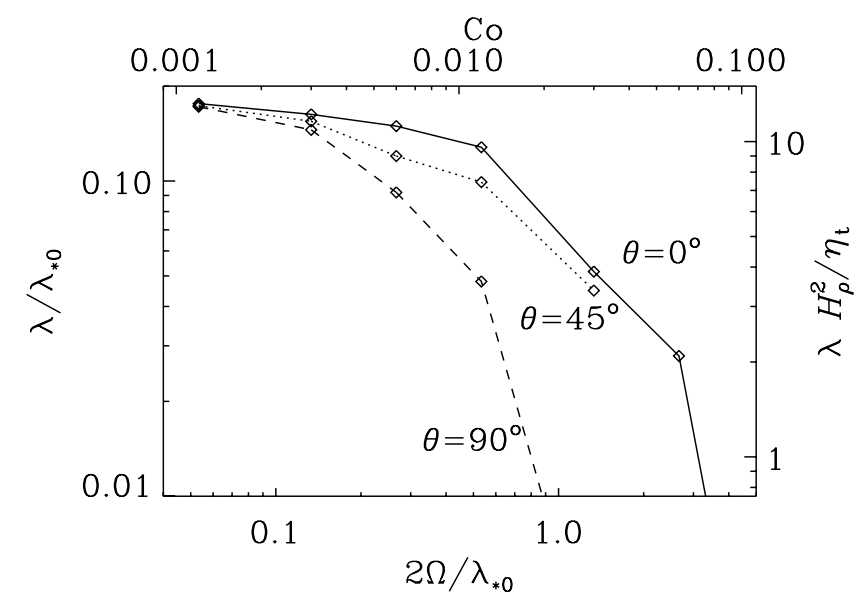

Fig. 4. Dependence of $\lambda / \lambda_{* 0}$ on $2 \Omega / \lambda_{* 0}$ for three values of $\theta$ for 3D simulations with $B_{0} / B_{\text {eq } 0}=0.05$.
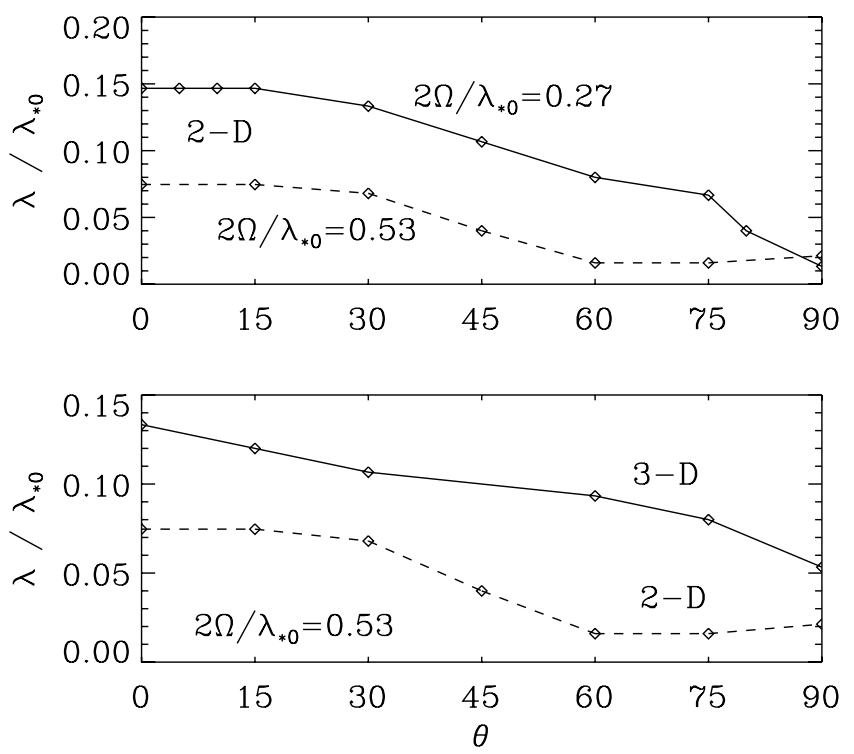

Fig. 5. Dependence of $\lambda / \lambda_{* 0}$ on $\theta$ for two values of $2 \Omega_{0} / \lambda_{* 0}$ in $2 \mathrm{D}$ (upper panel) and comparison of 2D and 3D cases (lower panel).

we have used $2 \mathrm{D}$ results, i.e. we restricted ourselves to solutions with $\partial / \partial y=0$, as was also done in Sect. 3. However, this is only an approximation of the fully 3D case. The usefulness of this restriction can be assessed by comparing 2D and 3D results; see the lower panel of Fig. 5. While the $\theta$ dependence is roughly similar in the 2D and 3D cases, the growth rates are by at least a factor of two lower in the 2D case.

To determine the oscillatory frequency, we consider the values of $\bar{U}_{y}\left(\boldsymbol{x}_{1}, \boldsymbol{t}\right)$ and $\bar{B}_{y}\left(\boldsymbol{x}_{1}, \boldsymbol{t}\right)$ at a fixed point $\boldsymbol{x}_{1}$ within the domain. As can be seen in Figs. 6 and 7, their frequency and amplitude depend on both $\Omega$ and $\theta$. The oscillations are not always harmonic ones, and can be irregular with variable periods, making the period determination more difficult. Nevertheless, the frequencies for $\bar{U}_{y}$ and $\bar{B}_{y}$ are similar over broad parameter ranges. For $\Omega_{0} / \lambda_{* 0}>0.25$ at $\theta=60^{\circ}$, NEMPI is no longer excited, but there are still oscillations in $\bar{U}_{y}\left(\boldsymbol{x}_{1}, \boldsymbol{t}\right)$, which must then have some other cause. We find a substantial variation in the amplitude for the maximum growth rate for $\Omega=0.01$ and $\Omega=0.02$. (The high frequency in $\bar{U}_{y}$ and $\bar{B}_{y}$ in Fig. 6 corresponds to a random small-amplitude change.) The frequency of the oscillations is very low at the poles, but it reaches a maximum at $\theta=45$ and decreases again toward the equator.

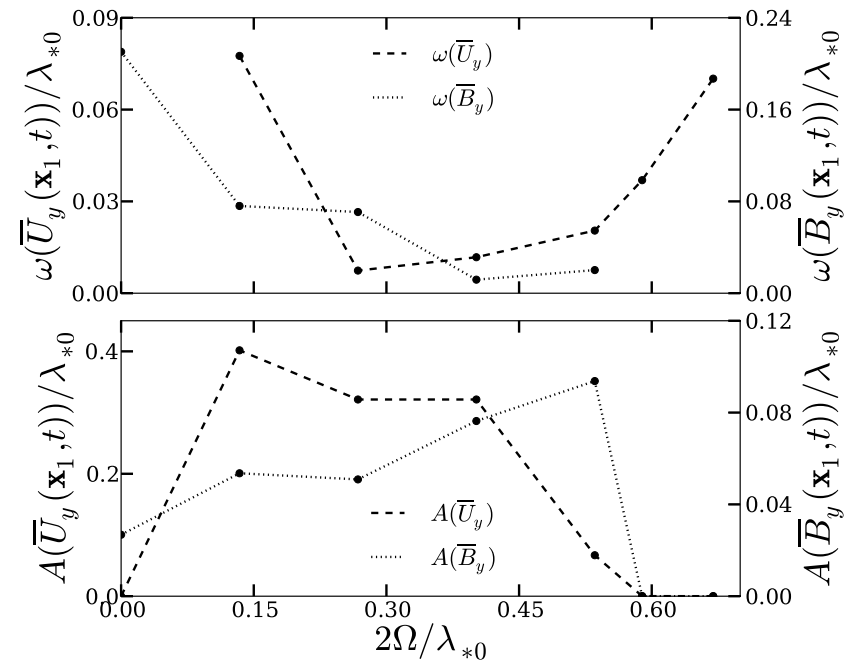

Fig. 6. Frequency and amplitude as a function of $\Omega$ for $\theta=60^{\circ}$ and $B_{0} / B_{\text {eq } 0}=0.1$ in the saturated regime.

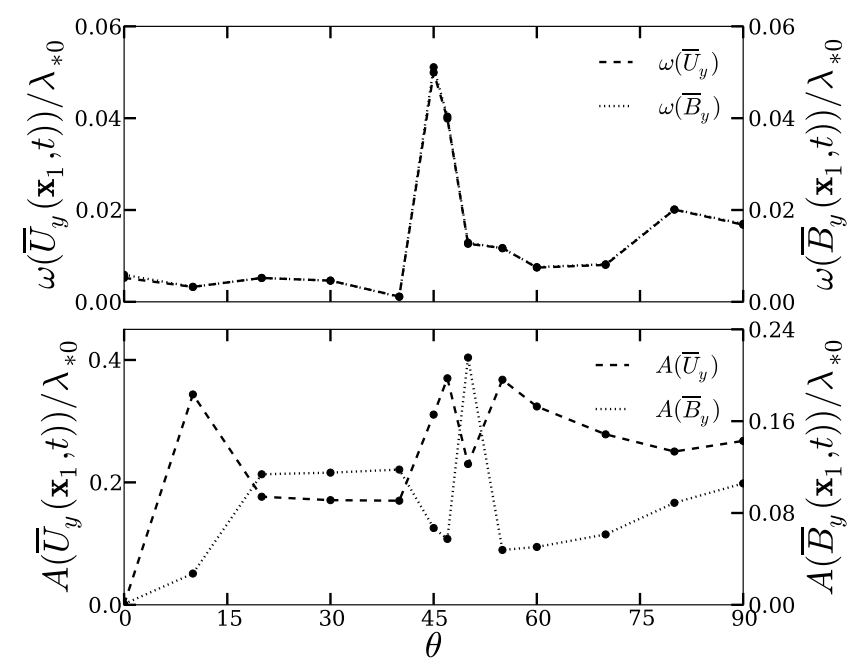

Fig. 7. Frequency and amplitude $\theta$ dependence for $\Omega=0.01$ and $B_{0} / B_{\text {eq } 0}=0.1$.

In summary, the oscillation frequency decreases (and the period increases) for faster rotation as the growth rate diminishes. Furthermore, the oscillation frequency is systematically lower at low latitudes (below $45^{\circ}$ ) and higher closer to the poles. We recall that these oscillations occur only in the nonlinear regime, so no meaningful comparison with linear theory is possible.

Given the combined presence of rotation and stratification, we expect the resulting velocity and magnetic fields to be helical. We plot relative kinetic, current, and cross helicities in the upper panel of Fig. 8. These are here abbreviated in terms of the function

$\mathcal{H}(\boldsymbol{p}, \boldsymbol{q})=\langle\boldsymbol{p} \cdot \boldsymbol{q}\rangle / \sqrt{\left\langle\boldsymbol{p}^{2}\right\rangle\left\langle\boldsymbol{q}^{2}\right\rangle}$,

where $\boldsymbol{p}$ and $\boldsymbol{q}$ are two arbitrary vectors. Here, $\langle\cdot\rangle$ denotes $x y$ averaging. The relative kinetic helicity, $\mathcal{H}(\overline{\boldsymbol{W}}, \overline{\boldsymbol{U}})$, where $\overline{\boldsymbol{W}}=$ $\boldsymbol{\nabla} \times \overline{\boldsymbol{U}}$ is the mean vorticity, varies between nearly +1 in the lower part and -1 in the upper part. This change of sign is familiar from laminar convection where upwellings expand to produce negative helicity in the upper parts, and downwellings also 

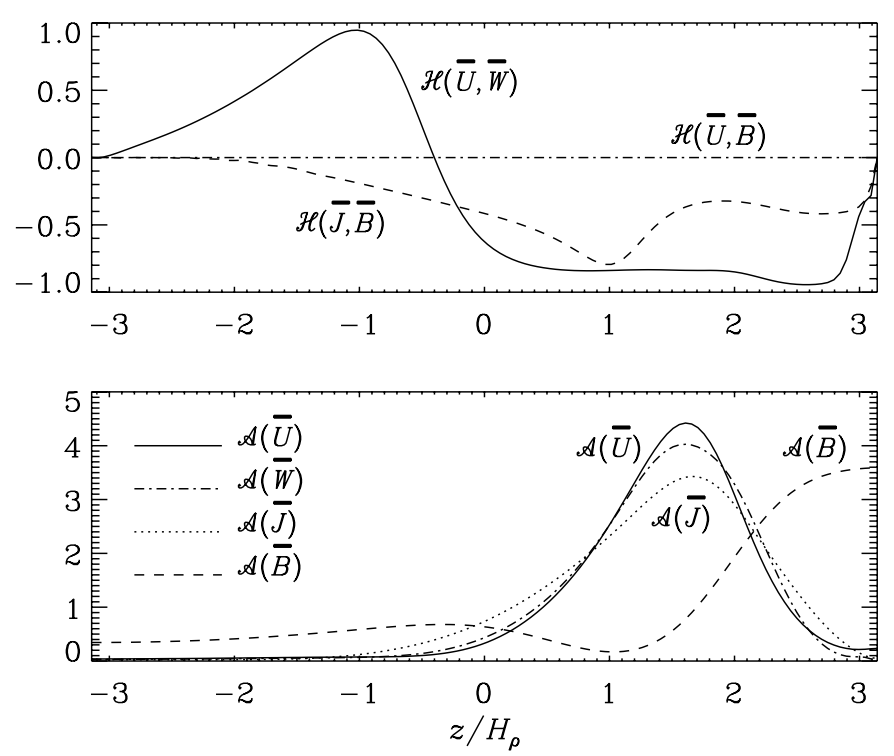

Fig. 8. Dependence of various relative helicities and relative amplitudes on $z$ for the case with $\theta=0^{\circ}$ and $\mathrm{Co}=0.03$.

expand as they hit the bottom of the domain (e.g. Brandenburg et al. 1990). However, in the lower part of the domain both $\bar{U}$ and $\bar{W}$ are relatively small, as can be seen by considering their relative amplitudes, $\mathcal{A}(\overline{\boldsymbol{U}})$ and $\mathcal{A}(\overline{\boldsymbol{W}})$, where

$\mathcal{A}(p)=\left\langle p^{2}\right\rangle /\left\langle\left\langle p^{2}\right\rangle\right\rangle$

with $\langle\langle\cdot\rangle\rangle$ being defined as volume averages.

It will be important to compare the present predictions of large-scale kinetic and magnetic helicity production with results from future DNS. One might expect differences between the two, because our current mean-field models ignore turbulent transport coefficients that are associated with helicity; see the discussion at the end of Kemel et al. (2012b).

We finally turn to the spatial structure of NEMPI. In Fig. 9 we compare $\bar{B}_{y}$ at different times and latitudes for the 2D runs. In the exponentially growing phase of NEMPI, the structures do not propagate (or move only very slowly). Traveling wave solutions occur mainly in a later stage of NEMPI, i.e., in the saturated regime. Next, we consider the 3D case. In Fig. 10 we show visualizations of the magnetic field on the periphery of the computational domain for four different times for $\theta=0$. Magnetic structures are inclined in the $x y$ plane. This is a direct result of rotation. As expected, the inclination is opposite for negative values of $\Omega$; see Fig. 11. The modulus of the inclination angle is about $30^{\circ}$, corresponding to 0.5 radians, which is not compatible with the value of $\mathrm{Co} \approx 0.03$, but it is closer to the value of $\Omega / \lambda_{* 0} \approx 0.65$. However, in this connection we should stress that we have imposed periodic boundary conditions in the $y$ direction, which means that the inclination angles only change in discrete steps. In the 2D runs, shown in Fig. 9, no inclination in the $x y$ plane is possible at all.

Returning to the case of positive values of $\Omega$, but $\theta \neq 0$, we note a slow migration of the magnetic pattern to the left (here for $\theta=45^{\circ}$ ), corresponding to poleward migration; see Fig. 12. Also the field is still tilted in the $x y$ plane. Finally, for $\theta=90^{\circ}$ we see that the pattern speed corresponds to prograde motion; see Fig. 13.

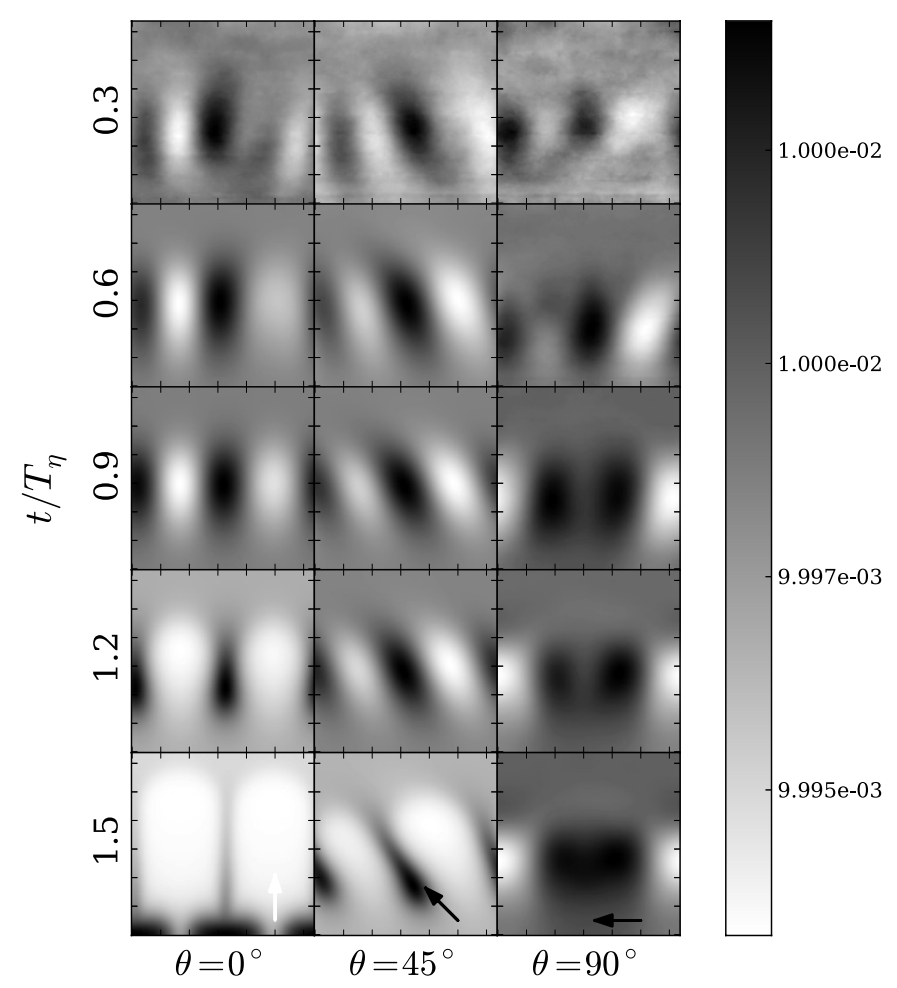

Fig. 9. Evolution of $\bar{B}_{y}$ in the $x z$ plane in a $2 \mathrm{D}$ simulation for $\Omega_{0}=0.01$ (corresponding to $\mathrm{Co}=0.006$ ) and $B_{0} / B_{\text {eq } 0}=0.1$ for $\theta=0^{\circ}, \theta=45^{\circ}$, and $\theta=90^{\circ}$ near the time when the instability saturates. The direction of $\boldsymbol{\Omega}$ is indicated in the last row.

\section{Conclusions}

Although the physical reality of NEMPI has recently been confirmed by direct numerical simulations, its potential role in producing large-scale magnetic structures in the Sun is still unclear. This paper begins the task of investigating its properties under conditions that are astrophysically important. Rotation is ubiquitous and clearly important in the Sun. The present work has now shown that the instability is suppressed already for rather slow rotation. This is rather surprising, because rotational effects normally become significant only when $\Omega$ is comparable to the inverse turnover time, which is defined here as $u_{\mathrm{rms}} k_{\mathrm{f}}$. The instability growth rate scale might explain this behaviour, since it is closer to the turbulent diffusive time than to the inverse turnover, which is faster by the square of the scale separation ratio (Brandenburg et al. 2011). However, our work now suggests that this is not quite right either and that the correct answer might be something in between. Indeed, we find here that growth rate and critical rotation rate are close to the parameter $\lambda_{* 0}=\beta_{\star} u_{\mathrm{rms}} / H_{\rho}$, which can be smaller than the aforementioned turnover time by a factor of 40 , although in solar convection, where $k_{\mathrm{f}} H_{\rho} \approx 2.4$ (Kemel et al. 2012d) and $\beta_{\star} \approx 0.23$ (Kemel et al. $2012 \mathrm{c}$ ), it is estimated to be only $\approx 10$ times smaller.

The suppression is strongest at the equator, where $\boldsymbol{\Omega}$ is perpendicular to the direction of the gravity field, i.e., $\boldsymbol{\Omega} \cdot \boldsymbol{g}=0$, and less strong at the poles where $\boldsymbol{\Omega}$ and $\boldsymbol{g}$ are either parallel (south pole) or antiparallel (north pole). In the absence of rotation, the mean magnetic field only varies in a plane that is normal to the direction of the imposed mean magnetic field, i.e., $\boldsymbol{k} \cdot \boldsymbol{B}_{0}=0$, where $\boldsymbol{k}$ stands for the wave vector of the resulting flow and magnetic field. However, in the presence of rotation the orientation of this plane changes such that now $\boldsymbol{k} \cdot\left(\boldsymbol{B}_{0}+\lambda_{* 0}^{-1} \boldsymbol{\Omega} \times \boldsymbol{B}_{0}\right)=0$. 

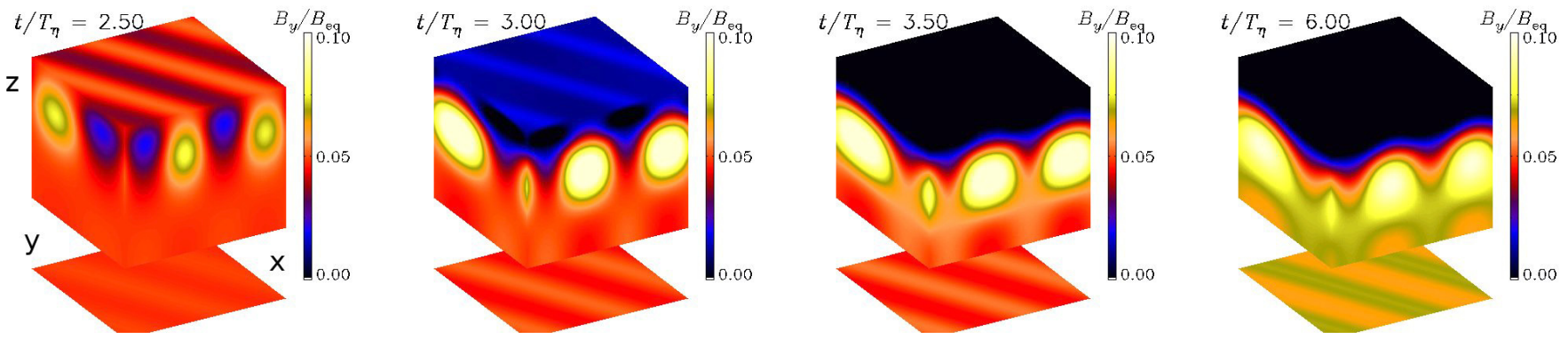

Fig. 10. Visualization of $B_{y}$ on the periphery of the computational domain for 4 times (normalized in terms of $T_{\eta}$ ) during the nonlinear stage of the instability for $\theta=0^{\circ}$ (corresponding to the north pole) and $\mathrm{Co}=0.03$, corresponding to $2 \Omega / \lambda_{* 0} \approx 1.3$. Time is here given in units of $T_{\eta}=\left(\eta_{\mathrm{t}} k_{1}^{2}\right)^{-1}$.
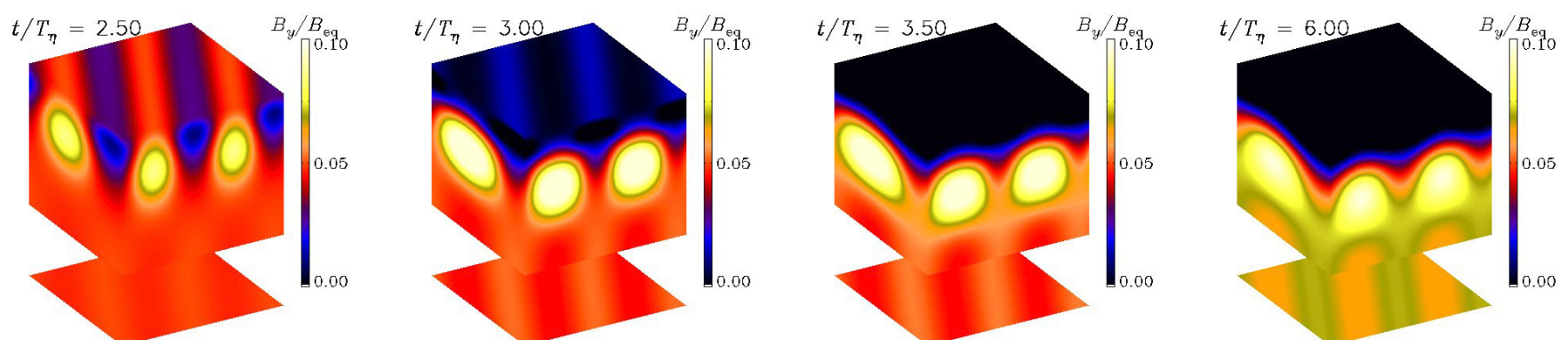

Fig. 11. Same as Fig. 10 , but for a negative value of $\Omega$, i.e., Co $=-0.03$, corresponding to $2 \Omega / \lambda_{* 0} \approx-1.3$.
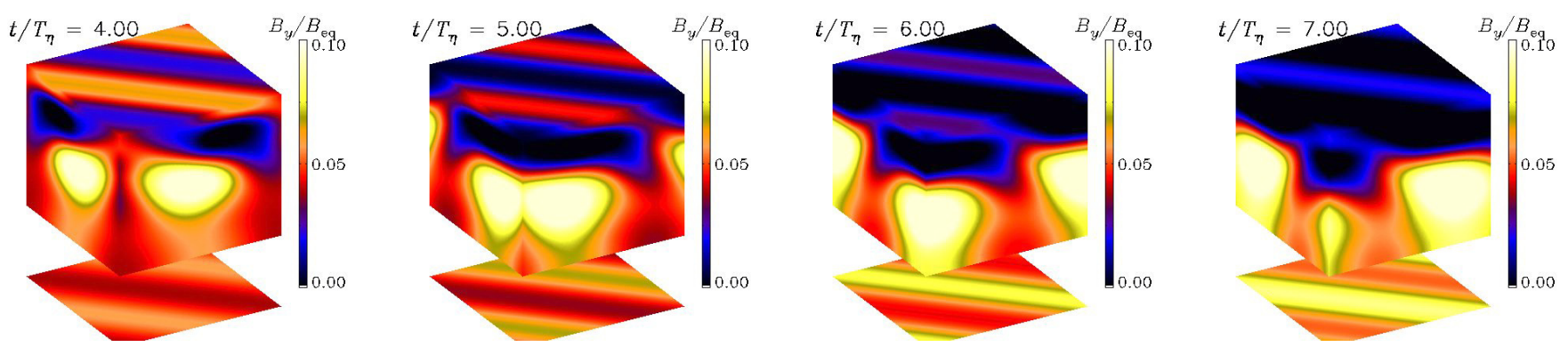

Fig. 12. Visualization of $B_{y}$ on the periphery of the computational domain for 4 times (normalized in terms of $T_{\eta}$ ) during the nonlinear stage of the instability for $\theta=45^{\circ}$ and $\mathrm{Co}=0.03$, corresponding to $2 \Omega / \lambda_{* 0} \approx 1.3$.
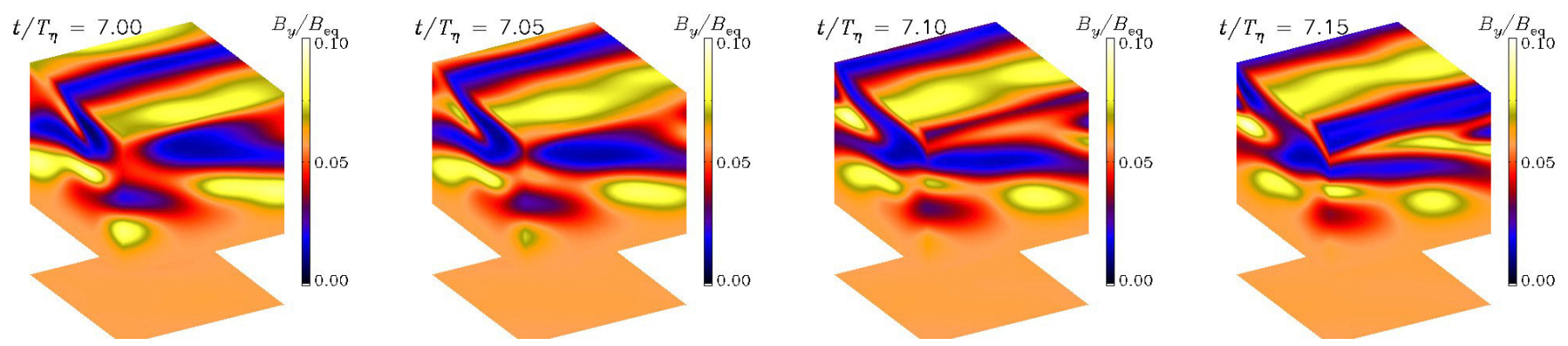

Fig. 13. Visualization of $B_{y}$ on the periphery of the computational domain for 4 times (normalized in terms of $T_{\eta}$ ) during the nonlinear stage of the instability for $\theta=90^{\circ}$ (corresponding to the equator) and Co $=0.013$, corresponding to $2 \Omega / \lambda_{* 0} \approx 0.5$.

At intermediate latitudes, i.e., when the angle spanned by $\boldsymbol{\Omega}$ and $\boldsymbol{g}$ is in the range of $0^{\circ}$ to $90^{\circ}$ colatitude, the magnetic field pattern propagates slowly in the negative $x$ direction, corresponding to poleward migration. The significance of this result is unclear. Had it been equatorward migration, one might have been tempted to associate this with the equatorward migration of the magnetic flux belts in the Sun from which sunspots emerge. On the other hand, at the equator this migration corresponds to prograde rotation, which is a clear effect seen in the Sun where magnetic tracers are seen to rotate faster than the ambient plasma, i.e., in the prograde direction (Gizon et al. 2003). Even sunspots rotate faster than the gas itself (Pulkkinen \& Tuominen 1998).

One of our goals for future work is to verify the present findings in direct numerical simulations. Such simulations would also allow us to determine new turbulent transport coefficients, 
similar to the $q_{\mathrm{p}}$ parameter invoked in the present study. Such additional parameters yield new effects, some of which could be important for applications to the Sun.

Finally, we end with a comment on the issue of scale separation. As discussed above, in solar mixing length theory, the correlation length of the turbulent eddies is expected to scale with the pressure scale height such that $k_{\mathrm{f}} H_{\rho}$ is constant and about 2.4 (Kemel et al. 2012d). Theoretical considerations have shown further that the growth rate of NEMPI is proportional to $k_{\mathrm{f}} H_{\rho}$. Since rotation is known to decrease the size of the turbulent eddies, i.e., to increase the value of $k_{\mathrm{f}}$, one might be tempted to speculate that rotation could even enhance the growth rate of NEMPI. However, in view of the present results, this now seems unlikely.

Acknowledgements. We thank the anonymous referee for making many useful suggestions that have improved the presentation of our results. Illa R. Losada was supported by $\mathrm{PhD}$ Grant "Beca de Investigación CajaCanarias para Postgraduaydos 2011". This work was supported in part by the European Research Council under the AstroDyn Research Project No. 227952, by the National Science Foundation under Grant No. NSF PHY05-51164 (AB), by EU COST Action MP0806, by the European Research Council under the Atmospheric Research Project No. 227915, and by a grant from the Government of the Russian Federation under contract No. 11.G34.31.0048 (NK, IR). We acknowledge the allocation of computing resources provided by the Swedish National Allocations Committee at the Center for Parallel Computers at the Royal Institute of Technology in Stockholm and the National Supercomputer Centers in Linköping.

\section{References}

Brandenburg, A. 2001, ApJ, 550, 824

Brandenburg, A., \& Dobler, W. 2002, Comp. Phys. Comm., 147, 471

Brandenburg, A., \& Subramanian, K. 2005, Phys. Rep., 417, 1

Brandenburg, A., Nordlund, Å., Pulkkinen, P., Stein, R. F., \& Tuominen, I. 1990, A\&A, 232, 277
Brandenburg, A., Kleeorin, N., \& Rogachevskii, I. 2010, Astron. Nachr., 331, 5 Brandenburg, A., Kemel, K., Kleeorin, N., Mitra, D., \& Rogachevskii, I. 2011, ApJ, 740, L50

Brandenburg, A., Kemel, K., Kleeorin, N., \& Rogachevskii, I. 2012, ApJ, 749, 179

Gizon, L., Duvall Jr, T. L., \& Schou, J. 2003, Nature, 421, 43

Käpylä, P. J., Brandenburg, A., Kleeorin, N., Mantere, M. J., \& Rogachevskii, I. 2012, MNRAS, 422, 2465

Kemel, K., Brandenburg, A., Kleeorin, N., \& Rogachevskii, I. 2012a, Astron. Nachr., 333, 95

Kemel, K., Brandenburg, A., Kleeorin, N., \& Rogachevskii, I. 2012b, Phys. Scr., in press [arXiv: 1208.0517]

Kemel, K., Brandenburg, A., Kleeorin, N., Mitra, D., \& Rogachevskii, I. 2012c, Sol. Phys., 280, 321

Kemel, K., Brandenburg, A., Kleeorin, N., Mitra, D., \& Rogachevskii, I. 2012d, Sol. Phys., DOI:10.1007/s11207-012-0031-8

Kleeorin, N., \& Rogachevskii, I. 1994, Phys. Rev. E, 50, 2716

Kleeorin, N. I., Rogachevskii, I. V., \& Ruzmaikin, A. A. 1989, Sov. Astron. Lett., 15,274

Kleeorin, N. I., Rogachevskii, I. V., \& Ruzmaikin, A. A. 1990, Sov. Phys. JETP, 70,878

Kleeorin, N., Mond, M., \& Rogachevskii, I. 1993, Phys. Fluids B, 5, 4128

Kleeorin, N., Mond, M., \& Rogachevskii, I. 1996, A\&A, 307, 293

Krause, F., \& Rädler, K.-H. 1980, Mean-field magnetohydrodynamics and dynamo theory (Oxford: Pergamon Press)

Moffatt, H. K. 1978, Magnetic field generation in electrically conducting fluids (Cambridge: Cambridge University Press)

Parker, E. N. 1966, ApJ, 145, 811

Parker, E.N. 1979, Cosmical magnetic fields (New York: Oxford University Press)

Pulkkinen, P., \& Tuominen, I. 1998, A\&A, 332, 748

Priest, E. R. 1982, Solar Magnetohydrodynamics (Dordrecht: D. Reidel Publ. Co.)

Rogachevskii, I., \& Kleeorin, N. 2007, Phys. Rev. E, 76, 056307

Sur, S., Brandenburg, A., \& Subramanian, K. 2008, MNRAS, 385, L15

Tserkovnikov, Y. A. 1960, Sov. Phys. Dokl., 5, 87

Vitense, E. 1953, Z. Astrophys., 32, 135

Zeldovich, Ya. B., Ruzmaikin, A. A., \& Sokoloff, D. D. 1983, Magnetic fields in astrophysics (New York: Gordon \& Breach) 